\title{
Expertengespräch: Ernährung und Nahrungsergänzung
}

Im täglichen Leben nimmt die Ernährung eine zentrale Rolle ein. Gesund zu essen gilt vielen Menschen als Ziel schlechthin, konstatieren unsere Interviewpartner, der TCM-Spezialist Beat Hornstein, der Naturheilkundler Bernhard Uehleke und der Ernährungswissenschafter Paolo Colombani. Doch bei den Essgewohnheiten regiert häufig der Irrtum: Vieles, was einen gesunden Ruf geniesst, ist in Wahrheit schädlich, und angeblich Ungesundes ist harmlos.

Welche Rolle spielt die Ernährung für die Gesundheit eines Menschen?

Hornstein: Sie ist essenziell. Neben den anderen Heilmethoden der TCM, also neben chinesischer Arzneitherapie, Akupunktur, Moxa, Schröpfen, Massagetechniken und Qi Gong/Tai Chi hat sie den wichtigsten Stellenwert. Allerdings steht ganz zuoberst das ärztliche Gespräch mit der Analyse der Lebensführung.

Uehleke: Die Ernährung hat eine ganz enorme Bedeutung. Der Darm ist sozusagen die nach innen gekehrte Wurzel unseres Organismus. Die Funktion des Darms ist sehr komplex; wir nehmen damit alle möglichen Nahrungsmittel auf und halten uns gleichzeitig die Gifte vom Leib. Trotz alledem ist dieser Darm für Krankheiten anfällig. In Mitteleuropa haben circa $30 \%$ der Bevölkerung funktionelle Magen-Darm-Beschwerden, vor allem Reizmagen und Reizdarm. Die Leidensgeschichten sind mitunter jahre- und jahrzehntelang. Für diese Patienten wäre es das Naheliegendste, mit ihrer Nahrung zu spielen, z.B. Rohkost wegzulassen. Wir haben aber im Rahmen einer Studie herausgefunden, dass die wenigsten dazu bereit sind, ihre Gewohnheiten zu verändern.

Colombani: Die Ernährung ist nur einer von vielen Einflüssen. Enorm viel wichtiger ist genügend Bewegung, und dass man nicht raucht. Werden diese beiden Faktoren in Studien sauber erfasst, sieht man nur einen sehr geringen Unterschied zwischen angeblich «guter» Ernährung - viele Früchte, wenig Fleisch usw. - und «schlechter» Ernährung - viel Fleisch, viele gesättigte Fette, wenig Gemüse und Früchte - in Bezug auf die Gesundheit. Das Problem ist, dass bislang nur in den wenigsten Studien die Bewegung sauber erfasst und so der Einfluss der Ernährung überschätzt wurde.

Wie gesund ernährt sich Ihrer Meinung nach die Allgemeinbevölkerung in der Schweiz?

Colombani: Das wissen wir nicht. Die Schweiz ist eines der wenigen Länder, wo das nicht systematisch erforscht ist. Mein Gefühl: So dramatisch schlecht kann es nicht sein. Viel schwerwiegender ist aber, dass sich die Leute nicht bewegen.

Uehleke: Speziell in der Schweiz ist man sehr gesundheitsbewusst. Man ernährt sich relativ vegetarisch, bewusst, vitaminreich. Nahrung ist auch etwas Teures. Die Qualität ist aber gut. Das Konzept der vollwertigen Ernährung aus naturheilkundlicher Sicht wird in der Schweiz sehr gut berücksichtigt.

Hornstein: Ich kann dazu sagen, dass sich in der Schweiz die Leute im Schnitt schlechter ernähren als meine
Patienten, die sich zusätzlich an den Prinzipien der altchinesischen Diätetik orientieren.

Was ist denn aus Ihrer Sicht eine gesunde Ernährung?

Hornstein: Es braucht dazu ein gewisses Verständnis für die Grundlagen der TCM. Krankheiten entstehen gemäss chinesischer Anschauung meistens aufgrund eines sog. Yang-Mangels. Yang bedeutet ursprünglich die sonnenbeschienene Seite eines Hügels - vereinfacht gesagt ist Yang Wärme oder Energie. Im Gegensatz dazu steht Yin für kälter und energieärmer. Auf dieser Basis sagen wir: Nahrungsmittel sollten möglichst warmer Natur sein, d.h. energiereich und leicht gekocht, nicht roh; vollwertig, aber nicht schwer verdaulich. Wir teilen die Nahrungsmittel aufgrund der Signaturenlehre in yang (warm) oder yin (kalt) ein. Warm sind z.B. Getreide, Reis oder Keime, ebenso Gewürze wie Paprika, Pfeffer, Ingwer, Knoblauch und Zwiebeln. Kalt sind Obst, Fisch und Milchprodukte. Auch Rohkost Salate, Tomaten, Gurken - und Kartoffeln sind kalt.

Die gelten alle als gesund ...

Hornstein: Das ist ja der Witz. In der Schulmedizin wird die Energetik nicht berücksichtigt. Wenn man das 


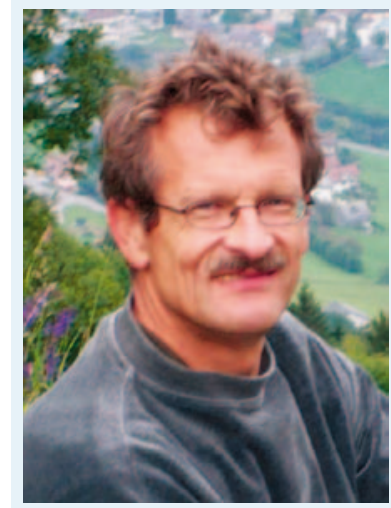

Dr. med. Dr. rer. nat. Bernhard Uehleke (Bernhard.Uehleke@usz.ch) ist wissenschaftlicher Mitarbeiter am Institut für Naturheilkunde am UniversitätsSpital Zürich. Er ist ausserdem Forschungsleiter der Abteilung Naturheilkunde an der Charité Berlin.

«Das Konzept der vollwertigen Ernährung aus naturheilkundlicher Sicht wird in der Schweiz sehr gut berücksichtigt.»

würde, hätte man einen Turbo, nur wird dieser leider nicht genutzt.

Colombani: Die Eckpfeiler einer guten Ernährung sind: nichts Extremes, keine maximalen oder minimalen Mengen eines bestimmten Nährstoffs, d.h. den goldenen Mittelweg finden. Das andere: Variation. Nicht ständig das Gleiche essen. Dadurch kommt man zu der Art und Weise, wie sich der Mensch immer schon ernährt hat. Die Natur hat nicht jederzeit Salat oder Eier oder Fleisch parat, das Angebot variiert. Das bringt den Stoffwechsel in Gang.

Was halten Sie von Ernährungsdogmen wie beispielsweise viel trinken?

Uehleke: Die Evidenzlage dieser Empfehlung ist sehr dürftig. Insofern ist diese Empfehlung in ihrer Absolutheit - zwei Liter pro Tag - kontraproduktiv und nicht einzuhalten. Ich selbst trinke mitunter wenig. Kneipp z.B. sagte, ein Pferd, das zu viel säuft, kann nicht mehr arbeiten. Anderseits gibt es Menschen, vor allem Frauen, die an der Grenze zur Dehydrierung stehen. Aber das Dauertrinken, auch von isotonischen Getränken, ist unsinnig. Der Mensch kann viele Stunden dürsten. Und vielleicht braucht unser System das zwischendurch, damit die Reguliermechanismen nicht verkümmern. Ein besserer Ansatz wäre: Das Trinken dem Körpergefühl überlassen, anstatt gegen die Instinkte literweise zu bechern.

Hornstein: Ich halte das ebenfalls für eine unsinnige Empfehlung. Irgendwie ist das Vieltrinken Mode geworden. Aus chinesischer Sicht ist es falsch. Man sollte grundsätzlich nach dem Durstgefühl gehen. Wenn man viel Wasser trinkt, muss die Niere dieses wieder ausscheiden; es braucht also Kraft. Die Kapazität der Niere ist aber begrenzt. Auch die Temperatur spielt eine Rolle. Im Sommer eisgekühlte Getränke hinunterzustürzen, ist $\mathrm{zu}$ vermeiden.

Herr Colombani, Sie haben kürzlich ein Buch unter dem Titel «Fette Irrtümer» veröffentlicht. Was ist denn so ein Ernährungsirrtum?

Colombani: Zum Beispiel die Schädlichkeit des Kaffees. Man hat ihn jahrzehntelang aufgrund eines einzelnen Stoffs, des Koffeins, verteufelt. Dabei hat man vernachlässigt, dass Kaffee aus unzähligen anderen Stoffen besteht, die mit dem Koffein wechselwirken und so dessen Wirkung beeinflussen und gar ausschalten können. Unterm Strich hat man für Kaffee meistens einen positiven Effekt.

\section{Gibt es weitere Mythen?}

Colombani: Die grosse Angst vor dem Fett - nicht nur bei der Normalbevölkerung, sondern auch bei den Fachleuten - und den Versuch, das Fett möglichst gegen null zu drücken. In den gängigen Ernährungsempfehlungen heisst es, der Fettanteil sollte 25-30\% des täglichen Energiebedarfs nicht überschreiten. Das ist eine extreme Empfehlung und aus wissenschaftlicher Sicht nicht zu halten. Weshalb die «Experten» der nationalen Ernährungsgesellschaften sie nicht schon längstens angepasst haben, ist mir ein Rätsel. Denn von der Empfehlung für den Fettanteil leitet sich diejenige für Kohlenhydrate $a b$, die mit $60 \%$ angegeben wird. Das ist Unfug! Eine dermassen kohlenhydratreiche Kost, das ist erwiesen, führt zu mehr Diabetes und Herz-KreislaufErkrankungen. Bei Urvölkern, die man untersucht hat, schwankt die Kohlenhydratzufuhr zwischen 22 und 40\% der täglichen Energiemenge, die Fettzufuhr zwischen 25 und $60 \%$.

\section{Wie soll ich den Fettkonsum} also handhaben?

Colombani: Indem Sie sich keinen Kopf machen und nicht versuchen, überall das Fett rauszuschneiden. $\mathrm{Ob}$ pflanzlich oder tierisch spielt dabei keine Rolle. Diese Auftrennung sehe ich wissenschaftlich ohnehin nicht begründet. Die überlieferte Aufteilung in «tierisch = gesättigt», "pflanzlich = ungesättigt» ist seit Jahren eine Fehlkommunikation. Selbst Schweineschmalz hat mehr ungesättigte Fettsäuren als gesättigte. Bei den tropischen Pflanzenfetten sieht es umgekehrt aus. Es war wohl ein Versuch, das zu vereinfachen, aber diese Vereinfachung ist nicht haltbar.

\section{Und wie sollte die tägliche Energiemenge Ihrer Ansicht nach zusammengesetzt sein?}

Colombani: Sinnvoll wären rund $40 \%$ Fett, $40 \%$ Kohlenhydrate, $20 \%$ Eiweiss.

Uehleke: Es gibt noch andere «Ernährungsweisheiten», denen man widersprechen muss, etwa dem Sprichwort: morgens wie ein Kaiser, mittags wie ein König und abends wie ein Bettler. 


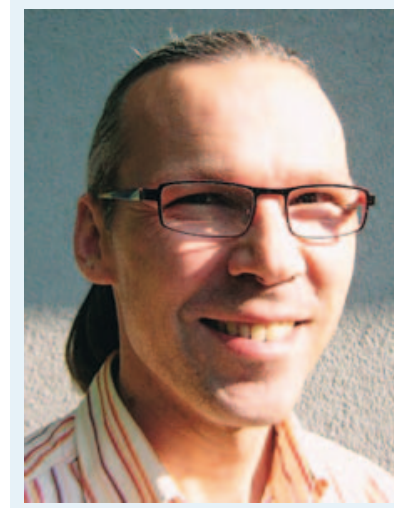

\section{Dr. med. Beat Hornstein}

(beat.hornstein@hin.ch) ist Arzt für Allgemeinmedizin und Traditionelle Chinesische Medizin mit eigener Praxis in Basel. Im Vorstand der Union schweizerischer komplementärmedizinischer Ärzteorganisationen vertritt er die Assoziation Schweizerischer Ärztegesellschaften für Akupunktur und Chinesische Medizin (ASA)

"Nahrungsmittel sollten möglichst warmer Natur sein, das heisst energiereich und leicht gekocht, nicht roh; vollwertig, aber nicht schwer verdaulich.»

Es mag ja einiges für sich haben, dass jemand, der mit Übergewicht $\mathrm{zu}$ kämpfen hat, auf abendliche fettreiche Mahlzeiten - die eher die Bildung von Fettdepots begünstigen - verzichtet. Andererseits ist es so, dass viele morgens einfach nichts vertragen. Sollen die ein schlechtes Gewissen haben und das Gefühl haben, ungesund zu leben? Das ist auch Quatsch. Wenn man das Ganze von der Evolution her betrachtet, so hatte der Urmensch seine Vorräte auch nicht immer neben seinem Bett zur Hand. Kinder zu quälen, bevor sie zur Schule gehen, halte ich für falsch. Und wenn man früh und schnell aufsteht, ist das System unter Umständen für eine reiche Nahrungsaufnahme noch gar nicht vorbereitet. Auch bei den vielen Zwischenmahlzeiten, die häufig empfohlen werden, muss man Zweifel anmelden. Fast pausenlos zu essen überfordert den Magen-Darm-Trakt, wie bei einer Spülmaschine, die ständig neu gestartet wird, obwohl das Programm noch läuft. Am Schluss kommt halbverdaute Nahrung raus.
Hornstein: Aus chinesischer Sicht hat jedoch das obige Sprichwort einen wahren Kern. Wer morgens nichts essen kann, hat schon eine geschwächte Verdauung.

\section{Kann eine gesunde Ernährung \\ Krankheiten vorbeugen? \\ Oder sogar heilen?}

Hornstein: Sowohl als auch. Die TCM betrachtet Nahrungsmittel als Heilmittel. Hühnerbrühe z.B. hat eine grosse Heilkraft: Sie ist energiereich, warm und enthält Kraft. Das Erkennen der Heilkraft beruht in der chinesischen Medizin auf der Analogielehre, also dem Entsprechungsdenken. Hühnerfleisch hat im Gegensatz zu Schweinefleisch sehr viel Yang. In meiner Praxis habe ich viele Beispiele von Patienten, die mit der «richtigen» Ernährung weniger Medikamente benötigen und schneller gesund werden. Wenn man sich hingegen kalt ernährt, entsteht ein Yang-Mangel. Das ist wie innerlich kalt abduschen. Wenn wir uns zu stark abkühlen, dann entsteht Krankheit.

Uehleke: Ernährungstherapie und Fasten sind eine der Säulen der klassischen Naturheilkunde. Das gilt sowohl für die Gesunderhaltung bzw. Prävention wie auch für die Heilung, etwa von entzündlichen Krankheiten wie Asthma oder Rheuma, durch eine fleischarme (genauer: Arachidonsäure-arme) Kost. Auch Gewürze wirken kurativ. Von den 300 monografierten Pflanzen in Europa deckt der grösste Teil das Thema Magen-Darm ab. Das gilt für aromatische Gewürze - Anis, Kümmel, Fenchel - genauso wie für Gewürze oder Bitterstoffe Enzian, Schnittlauch oder Petersilie.

\section{Was ist besser:}

\section{Rohkost oder Gekochtes?}

Colombani: Es ist besser abzuwechseln. Gewisse Nährstoffe gehen beim Kochen kaputt, andere werden besser verfügbar. Eine pauschale Antwort gibt es nicht. Durch die Abwechslung entsteht mehr Freude, mehr Geschmack und mehr Vielfalt.

Uehleke: Die Rohkost geniesst wegen der Vitamine hohes Ansehen, aber sie stellt hohe Anforderungen an unser Verdauungssystem. Nicht ohne Grund hat man das Kochen entwickelt, um die Nahrung leichter verdaulich $\mathrm{zu}$ machen. So wie wir ja in der Regel auch kaum rohes Fleisch essen, ist rohe pflanzliche Nahrung allein sehr belastend. Sehr viele Patienten haben deswegen Magen-Darm-Beschwerden, ohne sich dessen bewusst zu sein. Aber sie gilt halt als so gesund. Verkochen ist natürlich auch nichts; die Wahrheit liegt irgendwo in der Mitte.

Hornstein: Es kommt auf die Menge und die Gesamtbilanz an. Auch die Tageszeit und die Jahreszeit spielen eine Rolle; die ganze Energetik ist ja auch saisonal unterschiedlich. Zum Beispiel im Winter ergibt es Sinn, dreimal am Tag warm zu essen; im Sommer ist dies nicht so nötig. Unterm Strich sollte jedoch ein YangAufbau stattfinden. Man muss nicht chinesische Medizin studieren, um diese Grundsätze zu begreifen.

\section{Wie stehen Sie zum Fleischkonsum?}

Hornstein: Ist in der TCM grundsätzlich willkommen; es heisst ja, die Chinesen essen alles, was vier Beine hat. Grundsätzlich bedeutsam ist ein mässiger Konsum, eine gute Qualität und der Charakter: Hammelfleisch z.B. ist eher wärmend, Wild ebenfalls, Schwein eher kühlend. Ich persönlich finde eine artgerechte Haltung und den Respekt gegenüber den Tieren wichtig.

Colombani: Fleisch gehört zur menschlichen Ernährung, und zwar nachgewiesenermassen seit 2,5 Millionen Jahren oder noch länger. Noch wichtiger als die Frage der richtigen 


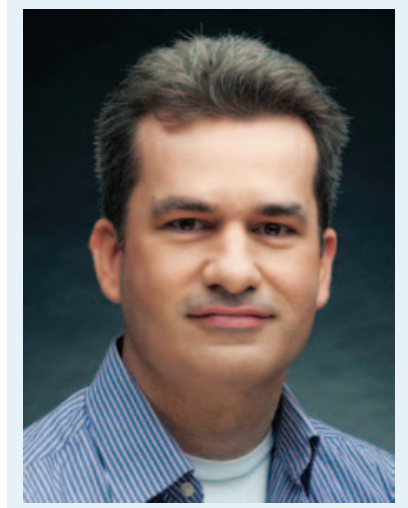

\section{Dr. Paolo Colombani}

(paolo-colombani@ethz.ch) ist Ernährungswissenschafter am Institut für Umweltentscheidungen (IED)/Departement Agrar- und Lebensmittelwissenschaften der Eidgenössischen Technischen Hochschule Zürich. Er ist Autor des kürzlich erschienenen Buchs «Fette Irrtümer» (Zürich, Orell Füssli, 2010; CHF 34,90).

«Die Ernährung ist nur einer von vielen Einflüssen aufdie Gesundheit. Enorm viel wichtiger ist zum Beispiel genügend Bewegung, und dass man nicht raucht.»

Menge ist auch hier die Variation: Statt immer das Gleiche sollten Geflügel, Schwein oder Rind immer wieder mal abwechselnd auf den Tisch kommen.

Uehleke: Der Fleischkonsum steigert generell die Entzündungsbereitschaft des Körpers. Für Menschen mit entzündlichen Erkrankungen - Asthma, Rheuma - wäre es ganz wichtig, mit einer fleischarmen, Omega-6- und Omega-3-fettsäurenreichen Ernährung gegenzusteuern. Dadurch kann man schon fast einen cortisonhaltigen Effekt erzielen.

\section{Sind Ihre Ernährungsempfehlungen vereinbar mit industriell ver- arbeiteten Lebensmitteln oder Convenience Food?}

Uehleke: Solche Lebensmittel haben natürlich viele psychologische Aspekte, die den Wert der Nahrung nicht mehr ins richtige Licht rücken. Man reisst eine Packung auf und haut rein. Natürliche Mechanismen werden da ausser Kraft gesetzt, insbesondere wenn noch Zusatzstoffe beigemischt sind, die unser Magen-Darm-System sozusagen täuschen. Ich erschrecke immer wieder, dass viele Patienten selber gar nicht kochen können. Deswegen denke ich, dass viele Menschen an ihren gesundheitlichen Problemen und an ihrem Übergewicht eine Mitschuld tragen. Und statt in der Schule allen möglichen Mist anzubieten, sollte man besser wieder Kochschulen einrichten.

Hornstein: Vereinbar sind sie schon. Aber man muss sehen: Hier geht es ums Geschäft; der Motor ist immer Geld verdienen. Je mehr hier dahintersteckt, desto weniger ist es energetisch sinnvoll. Je mehr Verarbeitungsschritte zwischen Urprodukt und dem «Food» liegen, desto energieärmer und «kühler» ist ein solches Nahrungsmittel. Manchmal muss man jedoch Kompromisse machen. Aber grundsätzlich gilt: Je weniger Konservierungsstoffe, künstliche Farbstoffe usw., desto geringer ist die biologische Belastung für den Körper.

Colombani: Da gibt es eine so grosse Vielfalt, dass man nicht pauschalisieren kann. Die Lebensmittelindustrie will Geld verdienen wie jede Industrie; das ist völlig normal. Bei den Trends muss man sagen: Das Marketing der Lebensmittelindustrie erachtet Dinge als sinnvoll, die gesamthaft nicht immer sinnvoll sind. Zum Beispiel die Anreicherung mit Mineralstoffen und Vitaminen aus der Haltung, dass man den Leuten damit einen Mehrwert verkauft. In einer halbwegs variierenden Ernährungsweise ist das nicht nötig. Ein sinnvoller Trend wäre: Möglichst wenig verarbeiten, nur das Notwendige, das Produkt muss nicht endlos haltbar sein, es darf wie in der Natur irgendwann kaputtgehen; und nicht zighunderte verschiedene Stoffe reinmixen, sondern möglichst wenig beimischen.

Welche Rolle spielen Nahrungsergänzungsmittel wie beispielsweise Vitalstoffe?

Colombani: Es gibt Umstände, die den Gebrauch von solchen Stoffen rechtfertigen. Eine besondere Situation bei Frauen ist die Schwangerschaft, wo man nicht weiss, ob sie wirklich genügend Vitamine oder Magnesium bekommen. Prophylaktisch den Stoffwechsel etwas zu unterstützen, finde ich hier sinnvoll, obwohl von der Natur nicht vorgesehen. Andere Stresssituationen gibt es im Spitzensport, bei Trainingsvorbereitungen etwa, Höhentraining usw.

Hornstein: Wer sich vollwertig ernähren kann, braucht keine Vitalstoffe oder Zusatzstoffe; das ist alles sekundär. Wem das nicht gelingt, kann davon vorübergehend profitieren. Es gibt Leute auf dem Weg zur Gesundung, und natürlich schwer Kranke, auf die das zutrifft. Chronisch Kranke z.B. tendieren dazu, sich falsch zu ernähren. Trotzdem ist dies für mich immer die zweite Wahl.

Uehleke: Der Gesunde in der Schweiz braucht dies nicht. In besonderen Stresssituationen können Vitalstoffe sinnvoll sein - in der Krebsnachsorge, Schwangerschaft, bei extremem Stress; da ist es mitunter angebracht, gewisse Vitamine und Mineralstoffe zusätzlich zuzuführen. Im Winter geraten zudem viele Leute - vor allem Ältere oder Berufstätige - in einen VitaminD-Mangel, weil sie sich im Sommer zuvor zu wenig der Sonne ausgesetzt haben. Wer sich jedoch im Sommer genügend Vitamin-D-Depots angelegt hat, kommt problemlos über den Winter.

Interview: Irène Dietschi 